\section{Electric Market and Utility Operation Terminology}

Ancillary services: Services that assist the grid operator in maintaining system balance. These include regulation and the contingency reserves: spinning, non-spinning, and in some regions, supplemental operating reserve.

Balancing area and Balancing Area Authority: The balancing area is a metered segment of the power system in which electrical balance is maintained by the balancing area authority. The fundamental balance that is required is that the total of all generation must equal the total of all loads, although this relationship is modified by electrical imports or exports into or out of the balancing area.

Base load generating plants: Typically coal or nuclear generating units, these plants are usually committed and dispatched at constant or near-constant levels, with minimum cycling. They are often the sources of lowest-cost of energy when run at very high capacity factors.

Commitment. Similar to Unit commitment: Commitment generally refers to the process of committing one or more units.

Contingency reserves: Reserve services that are sufficient to cover the unplanned trip (disconnect) of a large generator or transmission line and maintain system balance. Contingency reserves are generally split between spinning and non-spinning reserves, and are often based on the largest single hazard (generator or transmission capacity). Contingency events are big (many MW) and fast (within a few cycles). Forecast errors for variable generation are too slow to be contingency events.

Demand Response: Historically, utilities have controlled generation to assure reliability. Technically, controlling load can be as effective as controlling generation in matching net load. Demand response is the process using voluntarily offered (and compensated) load response as a bulk system reliability resource. Demand response currently provides half the contingency reserves in ERCOT as well as a major contribution in other regions.

Dispatching (economic dispatch): Once a plant has been committed, it can then be operated over a range of possible output levels. The economic dispatch process is a method by which system operators decide how much output should be scheduled from the plants that have been committed, and/or plants that can be started quickly and don't require a lengthy start-up or commitment process.
Energy imbalance service: A market service that provides for the management of unscheduled deviations in individual generator output or load consumption.

Flexibility: The ability of the generation fleet to change its output (ramp) rapidly, start and stop with short notice, and achieve a low minimum turn-down level.

Frequency response: Generation (and responsive load) capability to increase output (or reduce consumption) in response to a decline in system frequency and decrease output (or increase consumption) in response to an increase in system frequency. For generators, this requires governor response.

Generator trip: Electrical or mechanical malfunctions can sometimes cause a generator to fail. This is often called a "generator trip" and causes a contingency condition on the power system. A generator trip occurs very quickly, usually within a few cycles. Contingency reserves are held so that there is sufficient online generation that can replace the generator that tripped offline.

Intermediate (mid-merit) generating plants: Generating units that provide most or all of their energy during the day when energy demand increases. Intermediate generators can either turn off or cycle to a low minimum run level at night so they can match the diurnal demand patterns. Although some coal plants can provide this capability, it is more often gas, oil, or hydro plants that provide this service.

ISO (Independent System Operator): Responsible for maintaining system balance, reliability, and electricity market operation.

Load forecasting: Load forecasts are predictions of future demand. For normal operations, daily and weekly forecasts of the hour-by-hour demand are used to help develop generation schedules to ensure that sufficient quantities and types of generation are available when needed.

Load-interchange-generation balance: Aggregate load must be balanced with aggregate generation, accounting for energy imports and exports (also known as interchange) with neighboring systems.

LMP (Locational Marginal Price): This is the price of a unit of energy at a particular electrical location at a given time. LMPs are influenced by the nearby generation, load level, and transmission constraints and losses.

Minimum run (turn-down) level: Minimum level of output that can be provided from a generator. Different generators have different minimum run levels based in part on fuel source, plant design, or common use. 
Non-spinning reserve: Generation and responsive load that is off-line but can be fully responsive within 30 minutes. Contingency reserves include a component that is non-spinning. Non-spinning reserve can also be used to help manage solar variability and uncertainty for time-frames that exceed 10 minutes (load following)

On the margin: Power system operators dispatch generators based on cost and physical capabilities. Generators are dispatched sequentially from lowest to highest cost. The last generator to be dispatched at any point in time is referred to as the "marginal generator," and typically sets the market price for that market period.

Operating reserves: Combination of contingency reserves, regulation reserves, and sometimes load-following reserves. This term is not standard; different definitions are relatively widespread.

Peaking plants: These plants are often combustion turbines with low capital cost and high or very high fuel costs. They are therefore used sparingly, often only a handful of times a year during extreme peak periods of demand.

Ramp, ramp rate: Ramp refers to a change in generation output over some unit of time. Ramp rate describes the ability of a generating unit to changes its output, and is often measured in $\mathrm{MW} / \mathrm{min}$.

Rated Capacity: Maximum capacity of a generating unit.

Reactive supply and voltage control: The supply of dynamic reactive power, typically from generation, to control transmission system voltages.

Regulating reserves: Capacity that is devoted to providing the fast up and down balancing service. In the US regulating capacity is controlled by computers (automatic generation control, or AGC). Regulation duty can sometimes be procured in a market.

\section{RTO (Regional Transmission Organization): Responsible} for maintaining system balance, reliability, and electricity market operation.

Scheduling: This term refers to the general practice of ensuring that a generator is committed and available when needed. It also can refer to scheduling of imports or exports of energy into or out of a balancing area.
Spinning reserve: Generation and responsive load that is on-line, begins responding immediately, and is fully responsive within ten minutes. Part of contingency reserves must be spinning reserve.

System control: The central control function performed by the system operator that controls generation, demand response, and transmission assets to reliably and economically serve load.

Stack: The collection of available generators arranged in economic order. This term is often applied in the context of a specific type of system operation. For example, the dispatch stack refers to all generating units that are available for dispatch (have been committed or are quick-start units). The commitment stack refers to all generation units that have been committed or are available for commitment.

Sub-hourly energy markets: Electricity markets that operate on time steps of 5 minutes. Approximately $60 \%$ of all electricity in the U.S. is currently traded in sub-hourly markets, running at 5-minute intervals so that maximum flexibility can be obtained from the generation fleet.

Supplemental reserve: Generation and responsive load that is off-line but can be fully responsive within 10 minutes to replace spinning reserve that has been activated in response to a contingency or other power system need. Supplemental operating reserve is one of the contingency reserves. Turn-down capability: The minimum stable generation level that can be achieved by a generating unit.

Tie line: Individual BAAs have transmission connections to neighboring BAAs. The electrical configuration of these connections, called "tie lines" vary significantly. Part of the job of balancing supply and demand includes maintaining balance on the tie lines. Thus actual flows must equal scheduled flows, within a small allowable tolerance.

Unit: A single generator that may be part of a multiple-generator power plant.

Unit commitment: Is the process of starting up a generator so that boilers reach operating temperature and the plant is synchronized to the grid. This process can take many hours for a steam generator, depending on whether the plant is warm or hot from previous commitment.

Unloaded capacity: Generating capacity that is spinning and synchronized to the grid, but is not providing energy. asterater
EERE Information Center 1-877-EERE-INFO (1-877-337-3463) www.eere.energy.gov/informationcenter

Printed with a renewable-source ink on paper containing at least $50 \%$ wastepaper, including $10 \%$ post consumer waste.
DOE/GO-102011-3207 • May 2011 